\title{
Full Conjugate Heat Transfer Modelling for Steam Turbines in Transient Operations
}

\author{
M. Fadl \\ Department of Engineering Science, \\ University of Oxford, \\ Oxford OX2 OES, UK \\ P. Stein \\ GE Power, 5400 Baden, Switzerland \\ L. He \\ Department of Engineering Science, \\ University of Oxford, \\ Oxford OX2 0ES, UK
}

\begin{abstract}
Steam turbines are increasingly required to operate more flexibly, leading to a need for more detailed calculations and design considerations of the thermal conditions during transient operations. A major challenge in transient thermal designs is the time scale disparity. For the natural cooling of a turbine, the physical process is typically in hours or tens of hours, but on the other hand, the time step sizes typically usable tend to be very small, in seconds or sub-seconds, due to the numerical stability requirement for natural convection as often encountered.

The present paper reports a validation and demonstration study of a new loosely coupled conjugate heat transfer (CHT) approach developed for efficient and accurate simulations of steam turbine transient operations (Shut-down/ Natural cooling). The verification and validation have been carried out for a complex 3D geometry configuration with attributes of realistic but geometrically simplified full-scale steam turbines and compared with a well-established simplified working method backed up by experimental data. Further comparisons are made against a direct baseline CHT method. The present results demonstrate a generally good agreement with the simplified working method. The new loosely coupled method is shown to give a marked speed-up (by a factor of 240) compared to the baseline directly coupled method with the same accuracy. It thus offers the potential for significant improvement in predicting the long-duration transient natural convection conjugate heat transfer problems of steam turbines in terms of computational efficiency and accuracy.
\end{abstract}

\section{Introduction}

In the past decades steam turbines typically operated as base-load machines with constant operating conditions and a relatively low amount of start and stop cycles. In the meantime, combined cycle machines did show relatively stable operating regimes. Accordingly, the designs and their 
lifetime were based on large field experience of the OEM's fleet. This also enabled to develop and establish highly robust and fast design methods. In the most recent years, the market significantly changed, with the generic global target of decarbonization for environmental reasons, an increasing amount of more volatile contributors to the electric grid appeared and therefore demand for more flexible operation of the classical fossil machines [1] and [2]. In the mid-term future, the fossil machines will still exist and are required to balance the disadvantages of the current renewable technologies until they are fully established and provide stable energy at any time. Also for environmental reasons, the fossil machines have to show increased efficiencies. For the benefit of cycle efficiency during the past years, the steam turbine inlet temperatures have been further increased and show nowadays up to $620^{\circ} \mathrm{C}$, which will in near future be increased even further.

Additional to increasing steam turbine inlet temperatures, further efficiency increasing design options are developed like machines for double reheat cycles or further reduction of clearances to further reduce leakage losses. Besides operational flexibility and high turbine efficiencies, the current market is extremely competitive and therefore customer investments must be reduced as far as possible, but still maintaining a high machine value represented by high lifetime and high availability.

To keep the power plant operator investments low, the turbine designs have to be kept simple but still robust and the material grade has to be selected in a smart way to fulfil the above requirements but with the lowest possible degree of overdesigning the components. Such challenging designs can only be developed with the help of a large and long-term field experience in combination with development processes and tools which are accurate and enable deep insight into the physics of the design during operation.

For lifetime and availability, there is a little possibility of an accurate quantitative calculation for free convection phenomena in the cavities of heat engines, and primarily in gas and steam turbines, where their role is all the more important due to the temperature increase of the working medium. It is therefore not possible to make a proper evaluation of temperature distributions in units and components operating in the hottest zone, where temperature non-uniformities may lead to very considerable supplementary stresses, the permissible value of which is also determined by the temperature level. Moreover, in certain cases an underestimate of the effectiveness of convective heat removal may lead to structural complications due to the introduction of special artificial cooling, to avoid this, the thermal expansion behaviour must be understood for all occurring operating conditions, steady state as well as transient, hence start-up and shutdown [3]. 
Unsteady stresses in metal elements as well as temporal expansions (absolute and relative) of the turbine outer casing, inner casing, blade carriers, balance piston glands, outer shaft glands and turbine rotor during the start-up and shunt-down are very crucial to determine steam turbines lifetime. Here i.e. the temperature gradients between cold and warm condition are relevant which means a start from cold conditions consumes more turbine life than from hot condition [4]. Therefore, between shutdown and re-start the component temperatures become of interest. While typically the rotor with the first moving blade row groove forms the weakest part of the turbine and hence requires accurate prediction methods for the lifetime.

The formulation of the problem of free convection in steam turbines cavities is extremely complicated, mainly because of the diversity of cavity shapes and temperature boundary conditions. This leads to a very low efficiency of the experimental methods of investigation, since the results obtained may be applied only to a narrow range of problems. In fact, both the geometrical size of the cavity and the temperature conditions at its boundaries must be similar to those involved in the test.

A number of efforts have been made to develop modelling based methods for prediction of turbine shutdown and cooling processes where understanding the cooling-down thermo-fluid physics is important as it directly impacts thermal designs of the rotating and stationary components. Stein et.al [5] have shown that for high cycling turbines as used in solar applications, other components like the inner casing may also become critical with respect to lifetime and require accurate assessment methods. Born et.al [6] have shown that for steady state operating conditions even complex CFD/CHT calculations of nearly full steam turbines can be executed nowadays with high accuracy against validation measurements of full-scale machines. Mohr \& Ruffino [7] measured in a full-scale turbine the hot rotor surface temperature of a standard, combined cycle, intermediate pressure steam turbine using optical probes. The same data have been used by Marinescu et.al [8] for development of a full transient 2D method to calculate transient HP/IP steam turbine operations. Also, Marinescu has shown that the flow field can be captured even by using a Finite Element calculation method and treating the convective heat transfer as well as radiation by means of an adapted conductivity, which he called over-conductivity. This method was shown to be sufficiently precise and very robust against various measured designs [9]. In the first version, the over-conductivity function was calibrated against the measurements presented in [7]. In a later step, the computational domain was extended from 2D to 3D and split into regions to better distinguish the separate natural convection and radiation mechanisms. Here the equations to determine the over-conductivity for the separate mechanisms was taken from literature, and a later validation against measurements also confirmed a good agreement of the more generic method [10]. 
The above-described methods are shown to already enable with a certain accuracy and confidence temperature predictions for transient turbine operations. However, they rely on considerable simplifications in the calculation models and therefore require strong validations for each application. As consequence, the generic applicability and the large design changes are challenging for these types of methods.

High fidelity CFD for steam turbine applications shows the potential to massively increase the generic applicability of a design tool and this for much larger design changes. However, a CFD/CHT method, based on convective heat transfer analysis for determination of the heat transfer coefficient (HTC) of the flow field throughout turbine cavity with an assumption that HTC is largely invariant with respect to wall temperature. In such systems, generic spatiotemporal variation in the flow give rise to variation in the heat flux for a given fluid-solid temperature difference, which can be interpreted as spatiotemporal fluctuation of the instantaneous heat transfer coefficient.

For a natural convection problem of the present interest, the near-wall flow is dominantly driven by the wall temperature, making a HTC based approach even more difficult. This strong dependence of flow on the wall thermal condition in natural convection presents a strong case for pursuing conjugate heat transfer (CHT) solutions for coupled fluid-solid domains.

For general CHT method developments and applications, the time scale disparity between the fluid and solid parts as a fundamental feature must be recognized. The ratio between the two-time scales can be up to $10^{4}$ for gas turbine blades, as pointed out by He and Oldfield [10]. This time scale disparity presents a challenge for numerical solution methods adopted for the two domains as well as for the interface treatment between them. Fully coupled unsteady CHT simulations using the same discretization in space and time for both fluid and solid domains can be prohibitively expensive when a practical application such as a turbine flexible operation is to be dealt with. The problem would be difficult for URANS based CHT methods.

On the other hand, the time scale disparity can also serve as a justification for using a quasi-steady coupling approach for transient CHT, i.e. coupling an unsteady solid solver with a steady fluid solver at each time step. The quasi-steady based loosely coupled CHT methods have been actively developed and applied to forced convection heat transfer for turbine configurations and others, e.g. [11], [12], [13], [14]. For natural convection, there have been some attempts to use the direct coupled CHT solutions, e.g. [15] and [16], known to be computationally time-consuming. However, the present authors are not aware of any efforts of using a loosely coupled CHT approach for natural convection. 
A particularly relevant issue as has been observed is related to time step size allowable in natural convection simulations with common commercial codes (e.g. FLUENT, CFX and COMSOL). The numerical stability requirement tends to restrict the time step size to a very small value (typically subseconds), e.g. [17], [18], [19], [20], [21], [22], [23] and [26]. When a transient CHT solution for natural convection is pursued, this time step limit can be particularly restrictive, given the time scale disparity between the fluid and solid domain.

The present effort is chiefly motivated to investigate the applicability of a new URANS based loosely coupled CHT method for transient natural convection, relevant to steam turbine flexible operations. There are several related questions in this regard:

a) Validation and demonstration of the new loosely coupled procedure for solving transient conjugate heat transfer problems for natural convection, based on the recent efforts ([24] and [25]) and Multi-scale Time Integration for Transient Conjugate Heat Transfer, with an emphasis on a complex $3 D$ configuration and long-time scales typical of realistic steam turbine natural cooling processes.

b) Cross-validation between the present high-fidelity CFD and the over conductivity approach.

The coupling method is described in detail; the baseline solution results are then contrasted with the present source term based dual time stepping solver enabling a much larger time-step compared against others like direct coupled and over-conductivity.

\section{2-1. Baseline Flow Solver for Direct Coupled CHT}

The flow is governed by the 3D unsteady compressible flow equations, in a short form.

$$
\frac{\partial U}{\partial t}+R_{f}(U)=S_{b}(U)
$$

where $U$ is the vector of conservative variables and $R_{f}$ is the standard residual for forced convection computations. It is noted that the source term for the buoyancy effect $S_{b}$ is added and listed separately, as it is the treatment of this term which does have a very significant impact on the usable time step due to numerical stability. In all the present studies, regardless of numerical procedures, we adopt the full buoyancy term due to gravitational acceleration. Thus, the term is directly proportional to fluid density in line with the fully compressible flow model adopted.

For turbulence flows at high Rayleigh number conditions, the flow governing equations become Reynolds-averaged Navier-Stokes equations (RANS for steady flows or URANS for unsteady flows), 
more precisely the density weighted (Favre) time-averaged equations. In the present studies, the k- $\varepsilon$ two equation model is used for turbulence closure.

For unsteady flows, a pseudo time $\tau$ can be introduced for local iterations by time-marching at each physical step. A dual timing form with an implicit source term treatment should be in a form (for a physical time step $\mathrm{N}$ ):

$$
\left(\frac{\partial \mathbf{U}}{\partial \tau}\right)^{N, k}+\frac{1.5 \mathbf{U}^{N, k}-2 \mathbf{U}^{N-1}+0.5 U^{N-2}}{\Delta t}+R_{f}\left(\mathbf{U}^{N, k-1}\right)=S_{b}\left(U^{N, k}\right)
$$

where $\mathrm{k}$ is the index of the sub-iterations. When the sub-iterations converge, the pseudo time term vanishes, and the full 2nd order temporal approximation of the unsteady flow equation (Eq.1) fully recovers.

For a solid domain, the unsteady conduction equation governs the temperature field.

$$
\frac{\partial T}{\partial t}+R_{S}(T)=0
$$

Which is effectively in the same form as the unsteady fluid energy equation with zero velocity but also all fluid properties are replaced by those of the solid. The semi-discrete unsteady conduction equation in the dual timing form for time step $\mathrm{N}$ and sub-iteration $\mathrm{k}$ is:

$$
\left(\frac{\partial T}{\partial \tau}\right)^{N, k}+\frac{1.5 T^{N, k}-2 T^{N-1}+0.5 T^{N-2}}{\Delta t}+R_{S}\left(T^{N, k-1}\right)=0
$$

When a directly coupled CHT problem is solved, the physical conditions of temperature and heat flux continuity [20] between the fluid side (f) and solid side (s) are:

$$
\begin{aligned}
& q_{S}\left(T_{S}\right)^{N, k}=q_{f}\left(T_{f}\right)^{N, k} \\
& T_{S}^{N, k}=T_{f}^{N, k}
\end{aligned}
$$

A discretized form of Eq (5a), making use of Eq (5b), leads to an explicit expression of the wall temperature, so that the wall temperature is updated at each iteration, enabling solutions on both sides to be marched forward concurrently.

\section{2-2. Present Source Term-based Unsteady Flow Solver for Loosely Coupled CHT}

The present methodology has been implemented in the commercial code Fluent facilitating the UDF. Consider the fluid domain first, we now take a standard stand-alone fluid solution method for a steady natural convection problem as the baseline solver. Unsteady solutions are enabled 
completely through an extra source term explicitly added via UDF. The corresponding dual timing form of the fluid equation for physical time step $\mathrm{N}$ and sub-iteration step $\mathrm{k}$ becomes:

$$
\left(\frac{\partial \mathbf{U}}{\partial \tau}\right)^{k}+R_{f}\left(\mathbf{U}^{N, k-1}\right)=S_{b}\left(U^{N, k-1}\right)-\left(\frac{1.5 \mathbf{U}^{N, k-1}-2 \mathbf{U}^{N-1}+0.5 U^{N-2}}{\Delta t}\right)_{U D F}
$$

Note that both the physical temporal gradient term and the gravitational buoyancy term are in an explicit form, contributing to the balance of the unsteady flow equations when the pseudo-time term vanishes. A general dual timing formulation is known to be stable for large time steps due to the implicit backward difference for the physical temporal term, after Jameson [20]. It has also been widely used for URANS applications for unsteady turbomachinery flows with much larger physical time steps than that dictated by the CFL restricted numerical stability requirement (e.g. Arnone et al [21], He [23]). It is noted that the dual timing procedure may require the physical time step to be larger than that limited by CFL. This small-time step restriction, though not of concern for the present work, can be effectively overcome by simply limiting the pseudo time step [21].

As will be illustrated by the present results, this seemingly simple treatment of the physical temporal gradient completely as a source term, which is explicitly updated after each sub-iteration by UDF, is shown to have a clear and significant impact on the usable time step size for natural convection.

Similarly, for a solid domain, a standalone steady conduction solver is adopted. An unsteady conduction solution is also enabled by a source term by UDF, which is updated after each subiteration. The corresponding dual timing form for time step $\mathrm{N}$, and sub-iteration $\mathrm{k}$ is:

$$
\left(\frac{\partial T}{\partial \tau}\right)^{N, k}+R_{S}\left(T^{N, k-1}\right)=-\left(\frac{1.5 T^{N, k-1}-2 T^{N-1}+0.5 T^{N-2}}{\Delta t}\right)_{U D F}
$$

In a loosely coupled CHT system, the fluid solution, and the solid solution exchange information across the interface only after a number sub-iteration are completed. Each exchange between the two domains is called one CHT coupling cycle. A simple and stable interface treatment is to specify a wall temperature condition for the fluid domain and to specify a heat flux for the solid domain iteratively [25]. Within physical time step $\mathrm{N}$ and marching from CHT coupling cycle $\mathrm{i}$ to $\mathrm{i}+1$, the fluid domain first is solved with the Dirichlet condition provided from the solid solution, followed by a solid solution with the Neumann condition provided from the fluid one:

$$
\left(T_{f}\right)_{i+1}^{N}=\left(T_{S}\right)_{i}^{N}
$$




$$
\left(q_{S}\right)_{i+1}^{N}=\left(q_{f}\right)_{i+1}^{N}
$$

When the coupling cycles converge, Eqs (8) and (9) will be a direct manifestation of the physical temperature and flux continuity across the interface.

\section{2-3. Numerical modelling.}

A transient modelling including CHT of a simplified IP turbine has been carried out using highly efficient loosely coupled CHT method. Six domains are considered within the approach, which visible in Figure 1. Five metal domains (solid domains) are physically implemented into the model approach, these are the Thermal insulation, the outer casing, the inner casing, the bladed steam-path and the rotor. The thermal properties of the metals, such as specific heat capacity and thermal conductivity, are defined as a function of temperature. Furthermore, the fluid domain is modelled as Air using ideal gas model. For complexity reasons, the bladed steam-path was simplified to a solid modelling approach as presented in [6].

Both the fluid and solid meshes have been generated using tetrahedral meshes. The computational model contains about 138816 nodes in the fluid domain and 102856 nodes in the solid domains. General overview of computational grid in the solid sub-domains is presented in Table 1. Results have been checked to be not dependent on the grid chosen. The turbulence of the flow was modelled with k- $\varepsilon$ two equation model. The flow boundary layer was reproduced with a dimensionless wall distance $\mathrm{y}^{+}<10$. The calculations have been performed by using the commercial CFD solver ANSYS FLUENT.

A steady state condition at base load operation is used to define an initial metal and flow temperatures filed at the start of the transient cooling process, the thermal boundary condition at the external wall of the thermal insulation kept at $\mathrm{T}=295 \mathrm{~K}$ and $\mathrm{HTC}=10 \mathrm{~W} / \mathrm{m}^{2} \mathrm{~K}$. At the rotor end, also a fixed temperature and HTC is set to model the bearing impact on the heat fluxes. 


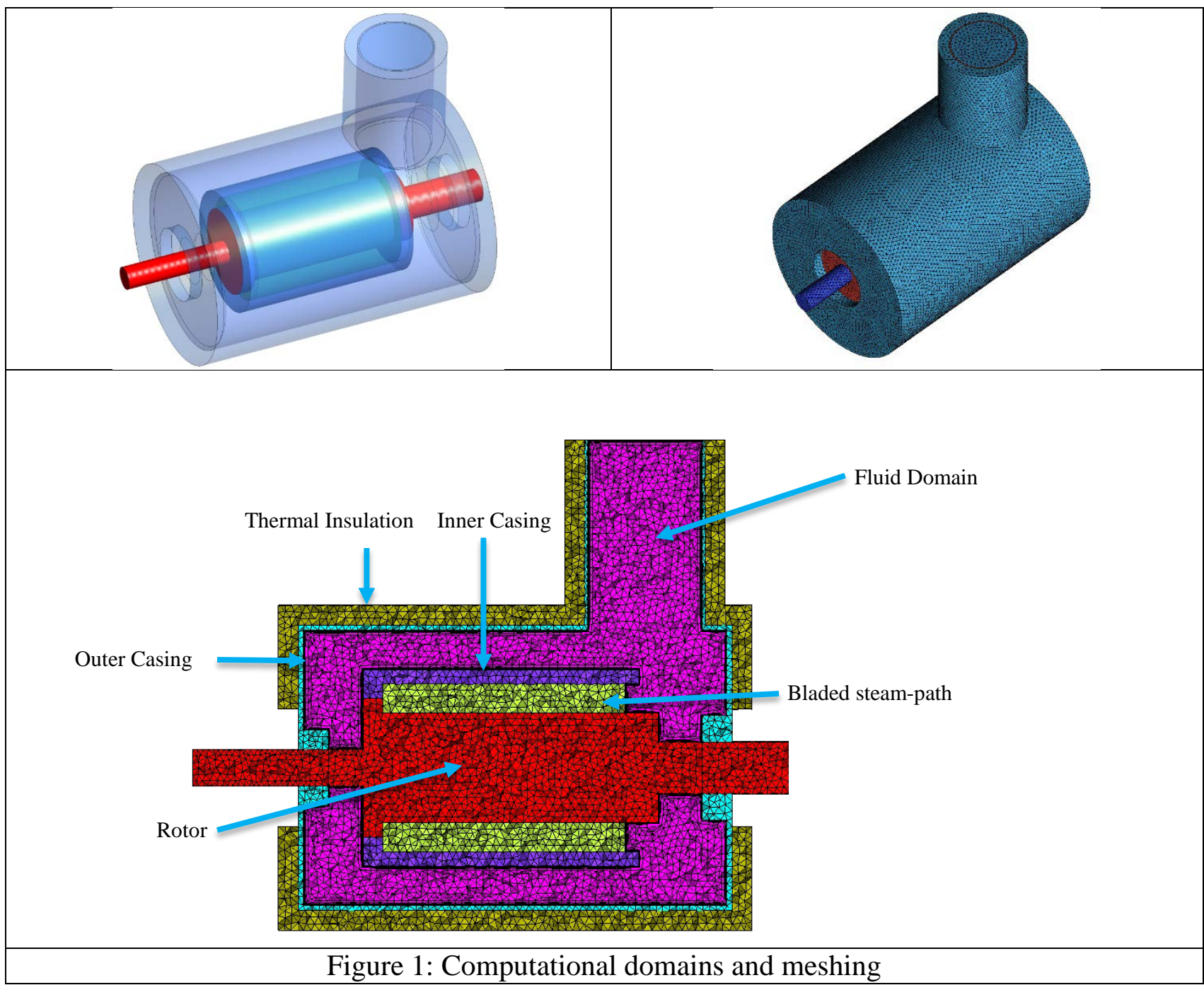

\begin{tabular}{|c|c|}
\hline \multicolumn{2}{|c|}{ Table 1. Computational grid in the solid domain } \\
\hline Blade path & 11745 \\
\hline Rotor & 12516 \\
\hline Outer casing & 22090 \\
\hline Inner casing & 46586 \\
\hline Insulation & 10464 \\
\hline
\end{tabular}

Figure 2 shows Temperature distribution along turbine cross section at the start of the natural cooling process. It is clear that there is a strong temperature gradient from the spiral inlet to last stage exit is visible, with the colder rear stage exit flow surrounding the cavity between inner- and outer casing. 

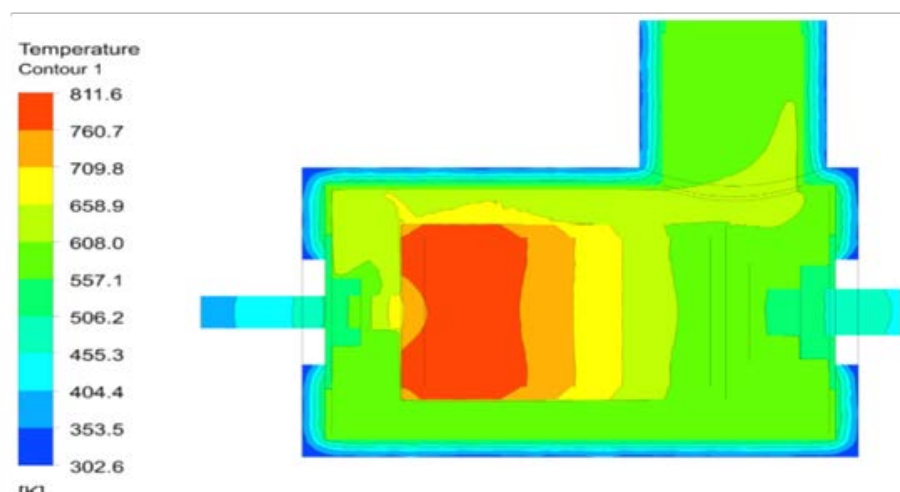

Figure 2: Temperature distribution along turbine cross section at the start of natural cooling

\section{Numerical validation of transient natural cooling.}

The validity of the novel loosely coupled analysis method was tested and validated by using two different approaches. In a first step, the loosely coupled analysis strategy was validated against a direct CHT solver. Because of the time-step limitations of a direct coupling in baseline solver and computational resources, the comparison between loosely coupled method and direct coupling during cooling down natural convection has been validated limited flow time, the dominating heat fluxes occur inside the metal parts with high-temperature gradients, i.e. the rotor and inner casing, where the high metal conductivity compared to a lower surface convection causes in a first step a temperature balancing along the solid parts. Later in time the heat fluxes through the surfaces become more relevant and dominate the temperature development over time. Nevertheless, this comparison is very valuable, as heat fluxes through the surfaces are still relevant. For larger overall process times, a second validation is required. Here the already existing and validated over-conductivity method [4, 6 and 9] was used to on the one hand side proof the loosely coupled methods ability to capture these long total times and secondly to show that the method is accurate.

As discussed in the introduction, common flow solvers in commercial codes have been previously shown to exhibit severe restrictions on time step size due to the numerical stability requirement of the solvers for natural convection. The previous effort by the authors have shown that [24], the severe time step restrictions of the baseline flow solutions can now be contrasted with the much larger time step sizes usable when the present source term based dual time stepping procedure is adopted.

Figure 3 shows the locations used for validation. Various locations were selected to show validity for all type of components, i.e. the technical critical ones like the rotor and the inner casing, but also the others like the outer casing and the rotor end parts as well as locations inside the fluid. This selection ensures that all types of heat fluxes are captured. 


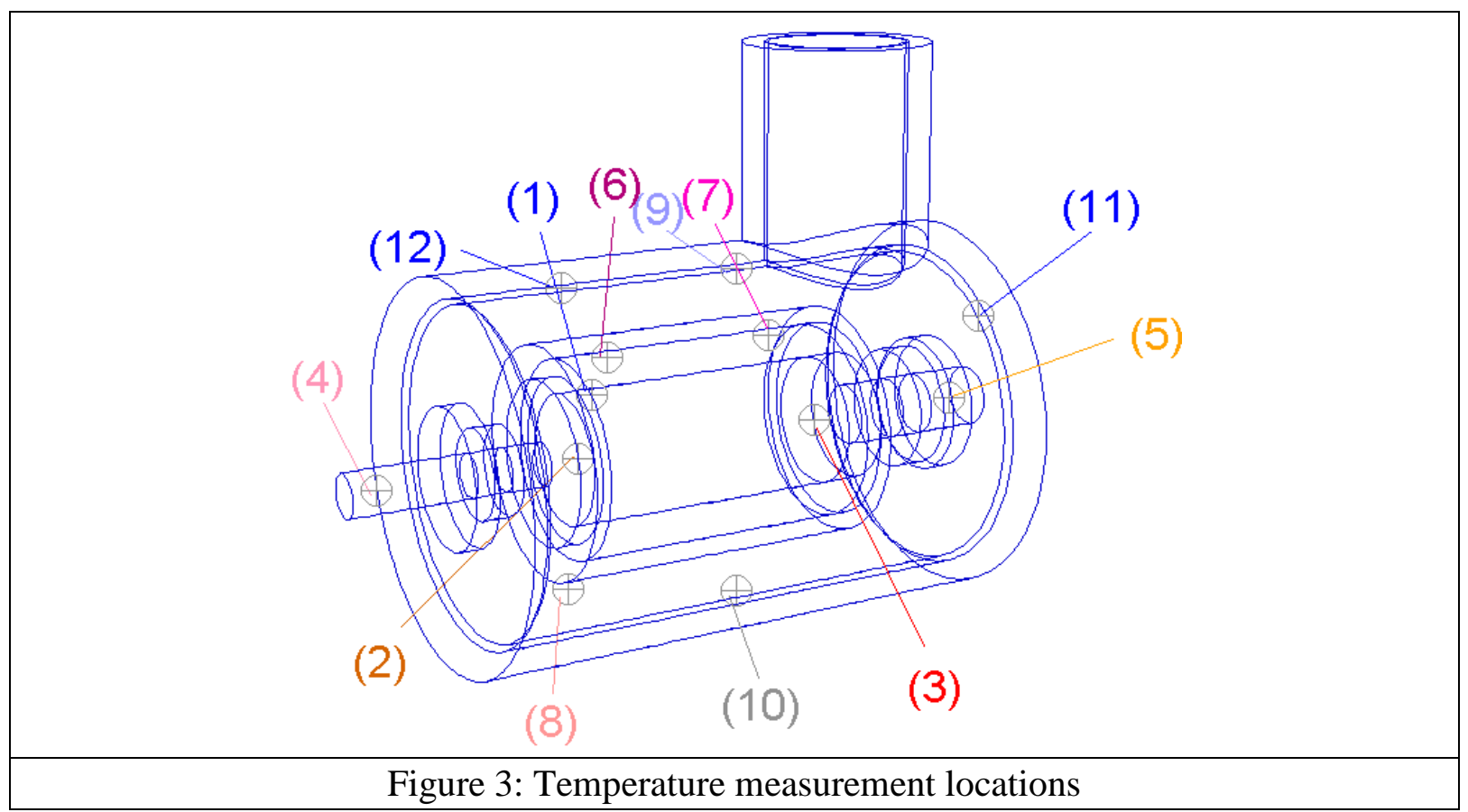

Figure 4 shows the development of the wall temperature at the various locations. The direct CHT simulation was performed using a small-time step size of $1 \mathrm{sec}$ which is the maximum time step allowed by numerical stability. A much large time step of $600 \mathrm{sec}$ was possible and used by the present solution. The comparison shows an excellent agreement between the present source term based solution and the direct solution, through with hugely different time step sizes. In Figures 5,6 and 7 , the rotor surface temperature, temperature and velocity in the middle section are compared at solution time of 2 and 5 hours. Clearly, both field solutions are also in good agreement.

In terms of computing effort, the direct solution for a 1-hour period requires 72000 iterations, using a physical time step of $1.0 \mathrm{sec}$ with 20 sub-iterations per time step. The present solution for the same physical time requires about 50 iterations. Therefore, for this case, the present solution is about 240 times more time efficient compared to the direct solution, simulated with the same flow solver.
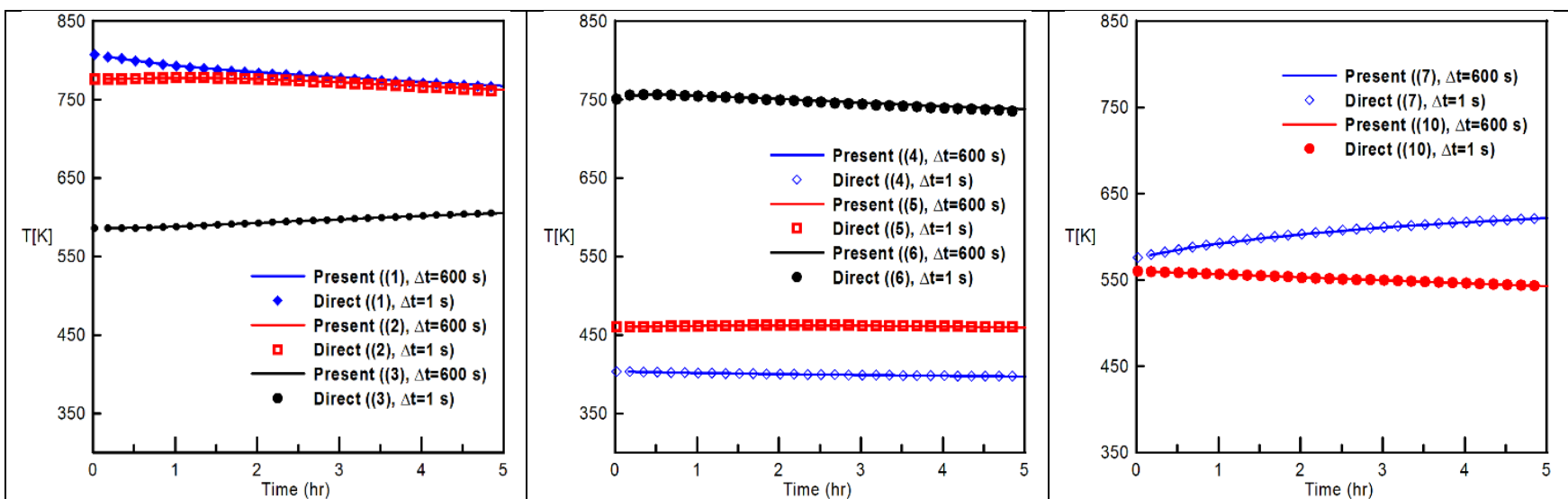

Figure 4: Comparison of temperature development during natural cooling between present solution $(\Delta \mathrm{t}=600 \mathrm{sec})$ and a direct solution $(\Delta \mathrm{t}=1 \mathrm{sec})$ 


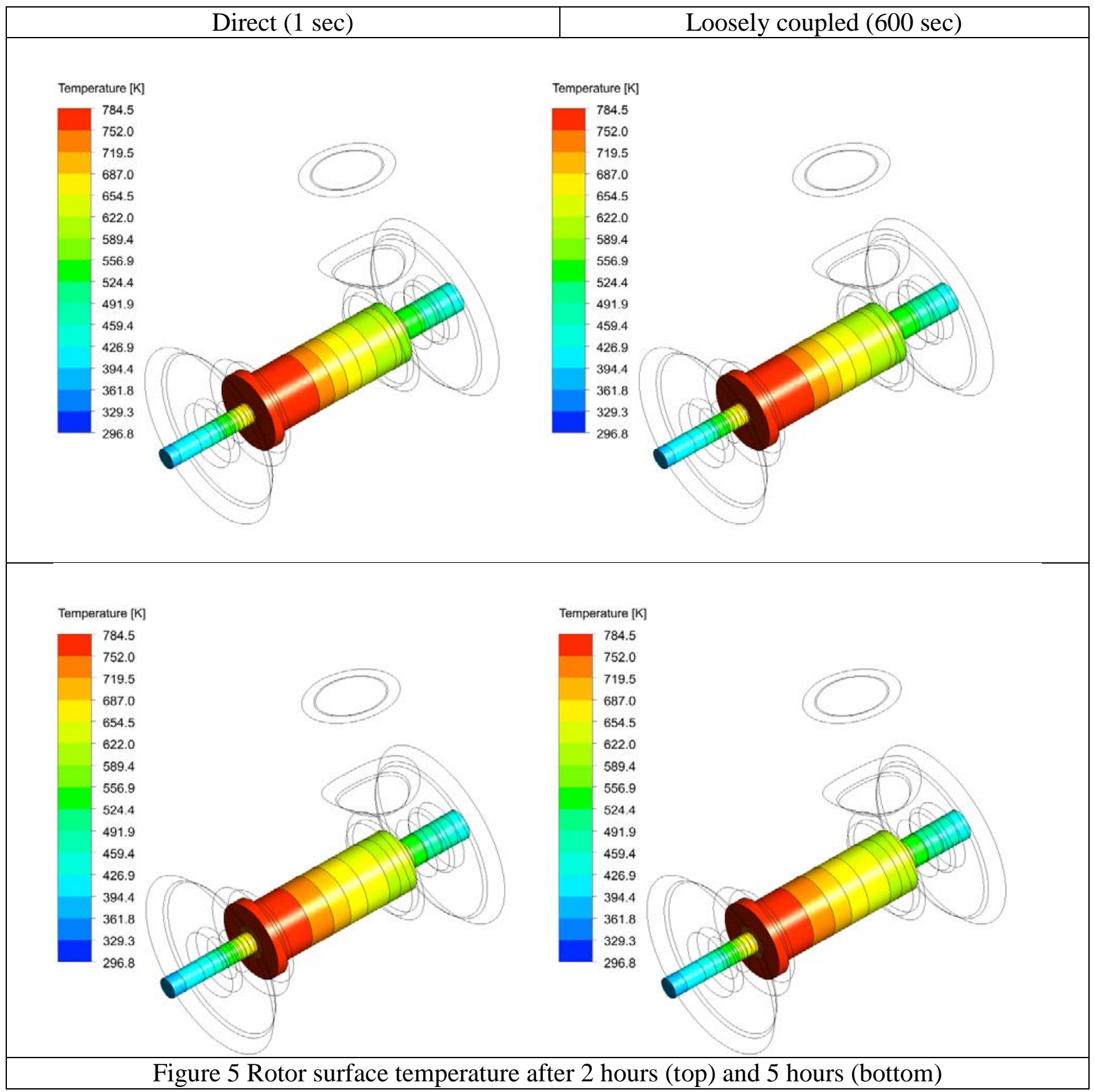




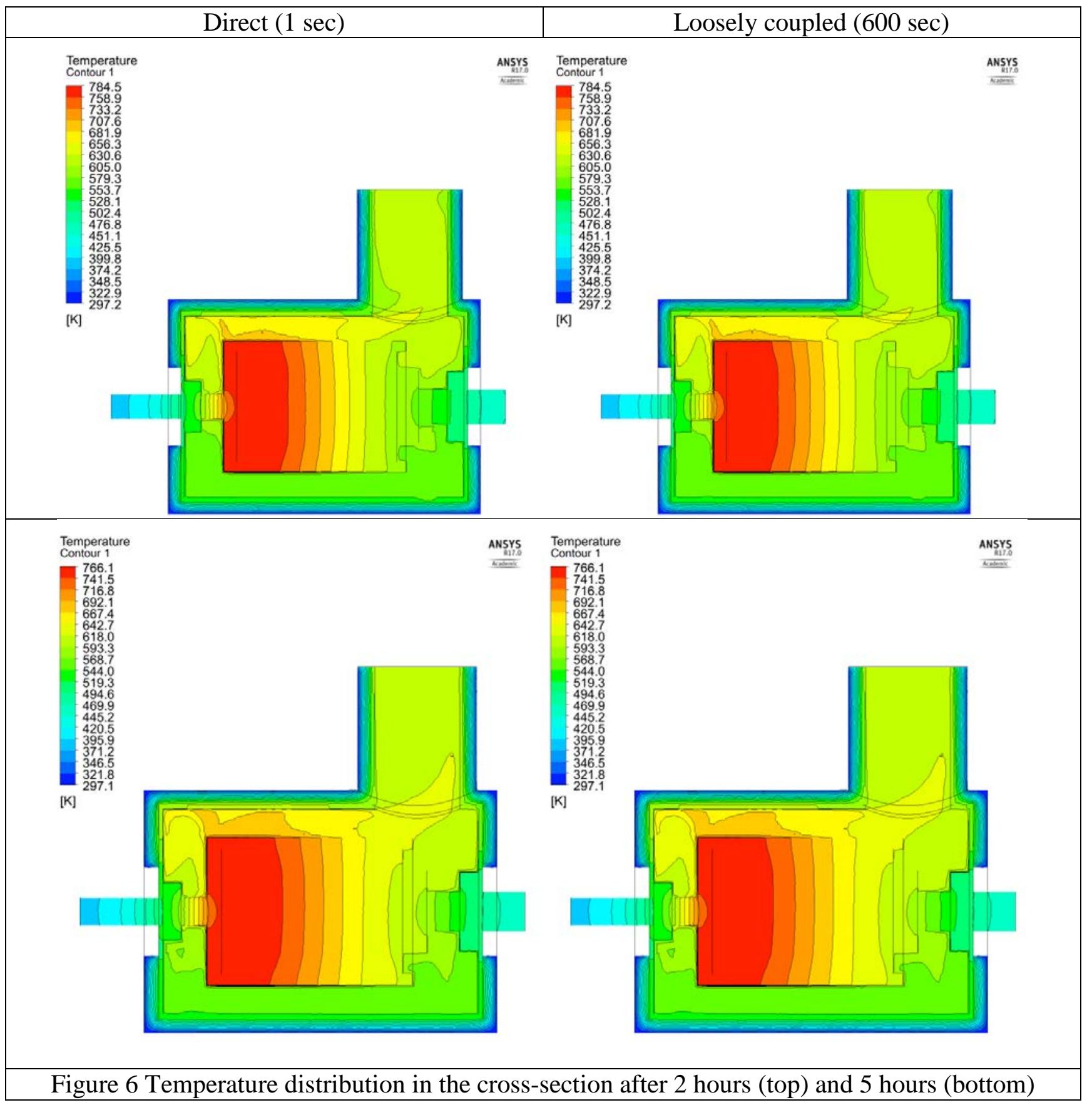




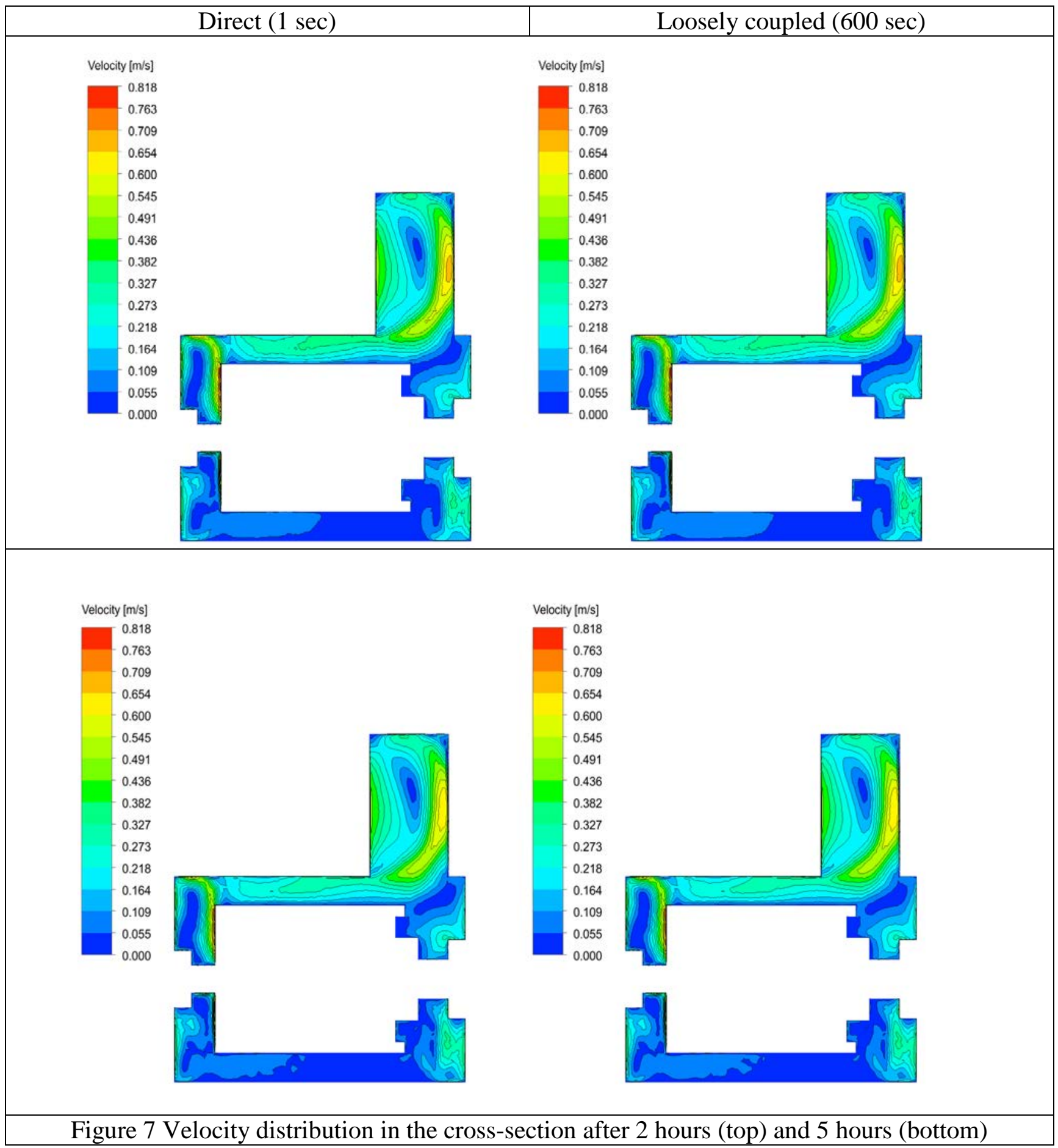


Validation of the temperature development at various locations for a total time of 100 hours and against the over-conductivity method is shown in Figure 8. At the mechanically loaded and with respect to the lifetime, more sensitive locations like rotor first blade row (locations 1 and 2) and the inner casing (location 6), both methods show a very good agreement and prove the applicability of the novel loosely coupled solution method. In other locations, i.e. the inner side of the outer casing (locations 8 and 10), a certain delay between the two methods is observed. Here i.e. the full strength of the novel method becomes visible. The temperature field inside the fluid domain of the over-conductivity model develops slowly from hot to cold and always forms a temperature gradient inside the fluid domain. This is somewhat artificial as in a real flow field with some turbulence, the fluid temperature is typically well mixed out and uniform. This is the advantage of solving the flow field as done with the present method, which avoids then these artificial time delays of the temperatures. Depending on the temperature development, this effect may be pronounced higher or lower. This deviation is also observed in the validation case of the over-conductivity model against measurements, presented in [6]. Therefore, it may be concluded that for the mechanical loaded and therefore lifetime critical locations the present method is accurate and on the top of this shows advantages with respect to the heat fluxes through the fluid, i.e. by appropriately considering the temperature mixing inside the fluid. Therefore, the temperature field throughout the whole domain including outer casing is improved. 

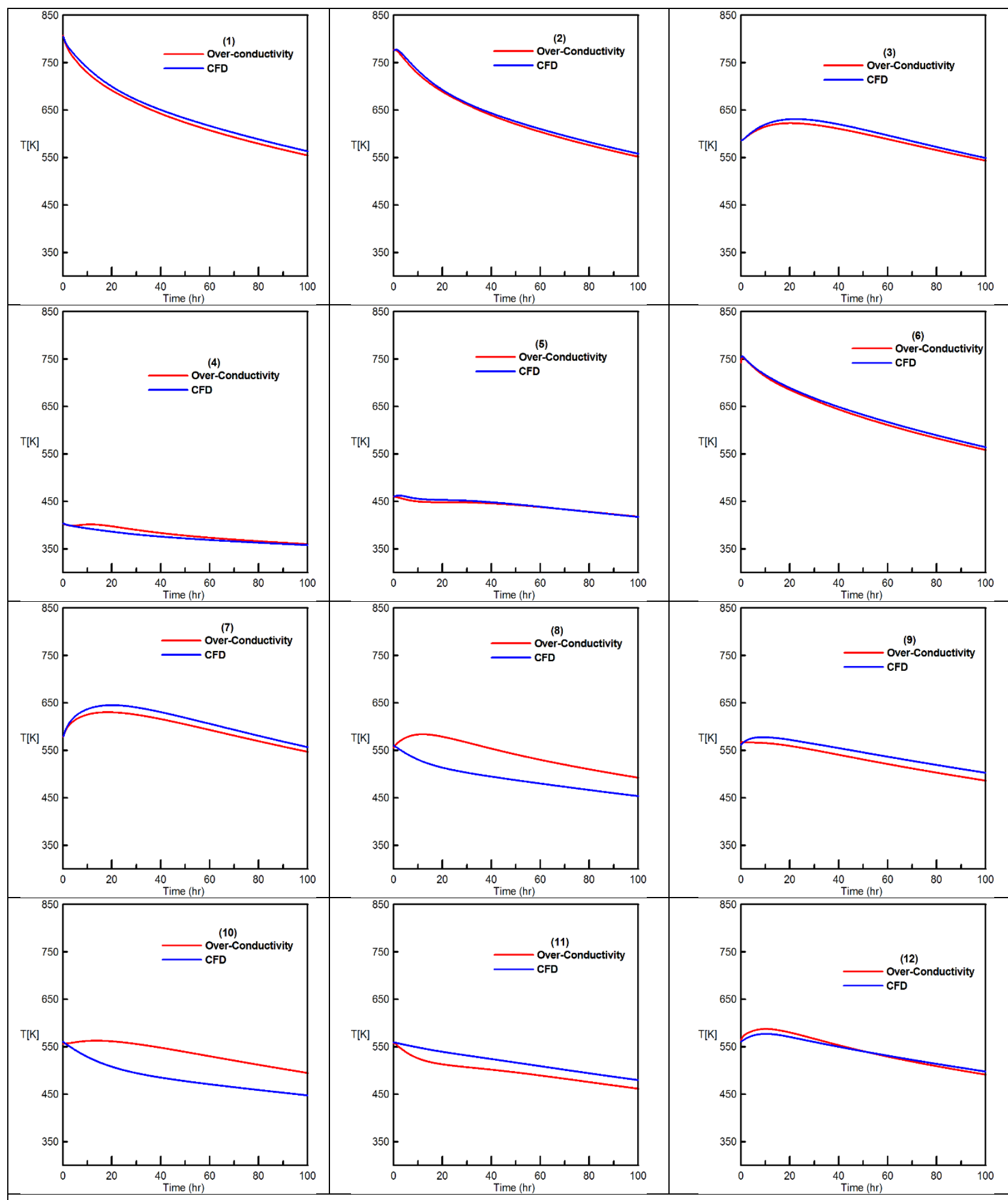

Figure 8 Temperature variation at different location, CFD versus over-conductivity 
Figure 9 shows a comparison between present method and over-conductivity for rotor surface temperature during natural cooling stage [25 and 100 hours]. The comparison shows very good agreement between the prediction and Over-conductivity model. It was found that the temperature differences in the inlet region are within $10-20^{\circ} \mathrm{C}$. This provides confidence on using loosely coupled CHT for simulation of the shutdown cool-down phase.

Also, a comparison of the temperature contour plots of a vertical cross-section between the two cases is reported in Figure 10. It is possible to see a very good agreement, demonstrating the validity of the ensemble-averaging principle as adopted and implemented in this work.

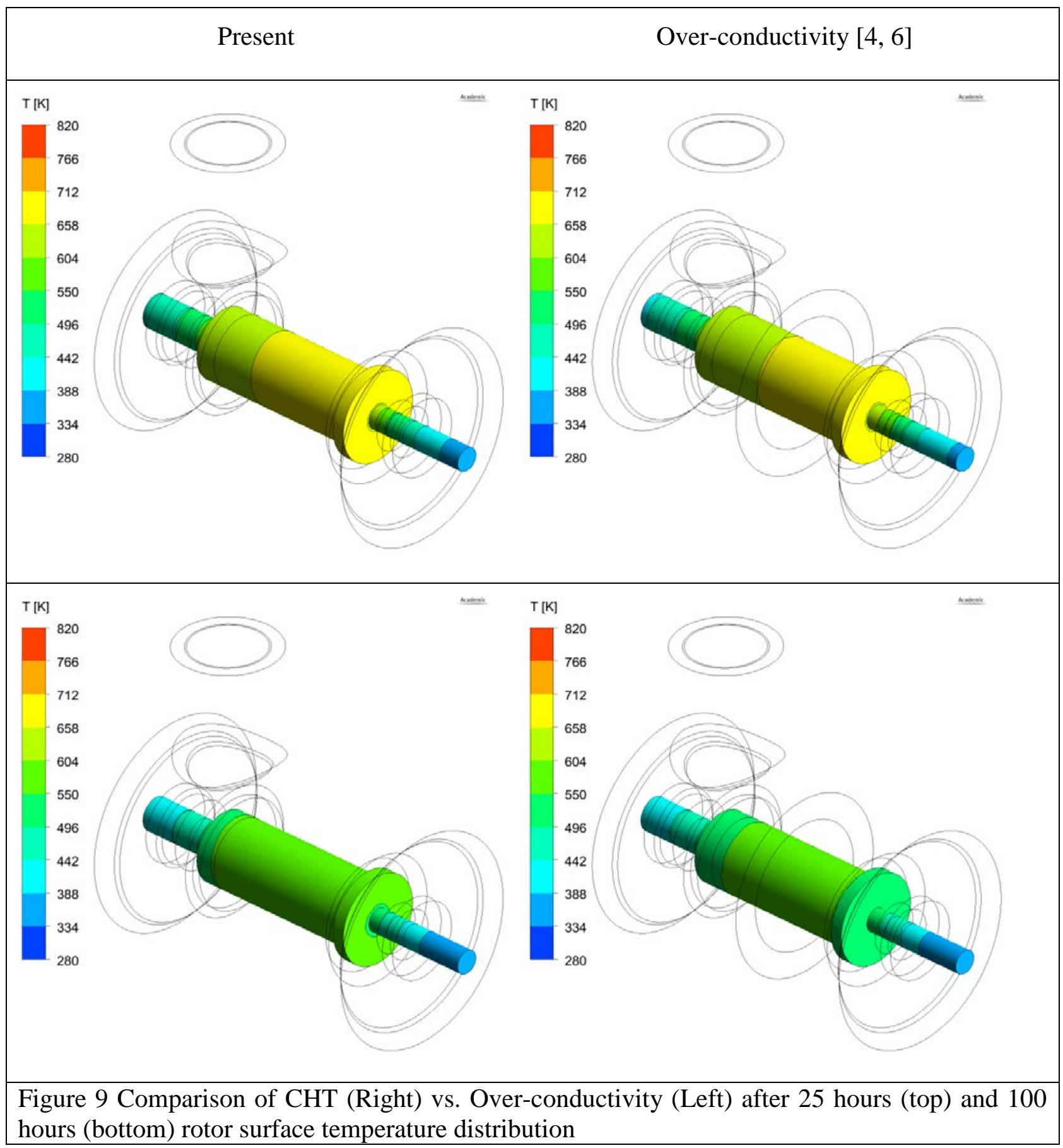




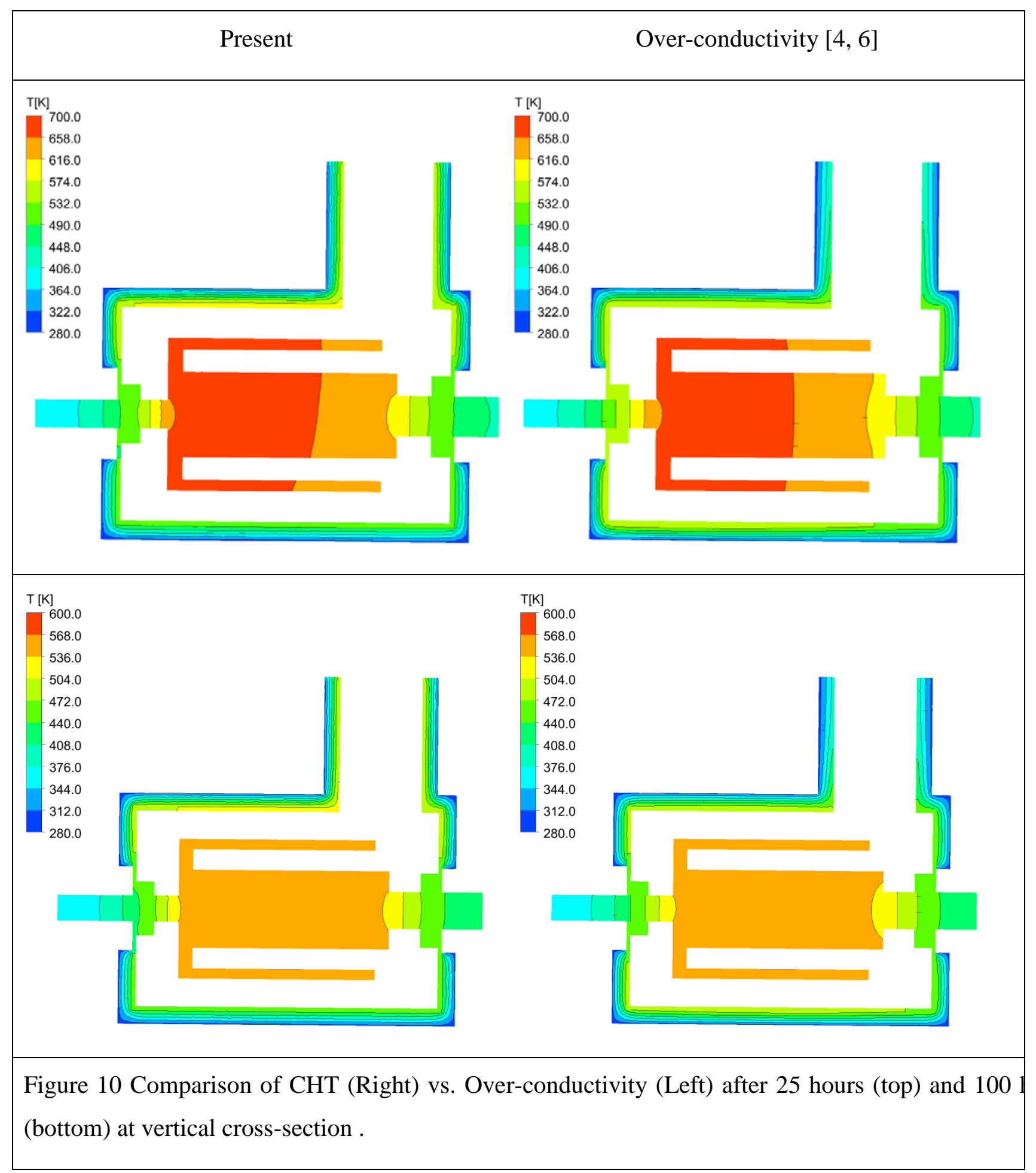




\section{Conclusion}

This paper focuses on the validation of a newly developed numerical model based on CHT/CFD calculations against a close-to-real configuration of an intermediate pressure steam turbine for the highly complex natural cooling process. The aim is to show the feasibility of this novel method with respect to analyzing real-world problems. The validation itself was performed against a full direct coupled CHT/CFD computation for a limited time of 5 hours of turbine natural cooling. The presented method was shown to be about 240 times more time efficient than a direct coupled solution. Furthermore, a validation was executed against an already developed and successfully validated calculation process using a so-called over-conductivity method. Both validations were done with a simplified turbine model, as the computational effort is still quite high, this work was targeted to demonstrate a generic feasibility rather than presenting a fully developed design tool.

The results show a highly promising method with a great accuracy for the present validation cases. In the mechanically most highly loaded and therefore design relevant regions, both methods show a very good agreement, even after 100 hours of natural cooling. In other regions, the present novel method demonstrates its full strength as it can overcome limitations of the over-conductivity method due to the inherent empiricism that the simple working method has been based on. Therefore, the presented full CHT modelling method of the turbine is shown to be feasible and accurate with respect to transient natural convection. It can successfully handle complex cases like real turbine designs for very large time step sizes and therefore long overall time periods. With this approach, the full turbine operation cycle may be accurately captured with full flow field calculations from start-up to baseload and shut-down for many tens of hours.

\section{Acknowledgments}

The present work is sponsored by UK Engineering and Physical Science Research Council (EPSRC) and GE Power.

\section{Nomenclature}

CFD Computational fluid dynamics

CHT Conjugated heat transfer

CFL Courant-Friedrichs-Lewy condition

HTC Convective heat transfer coefficient $\left[\mathrm{W} / \mathrm{m}^{2} \mathrm{~K}\right]$

q Wall heat flux $\left[\mathrm{W} / \mathrm{m}^{2}\right]$

R Standard residual

$\mathrm{S}_{\mathrm{b}} \quad$ Buoyance source term 


\begin{tabular}{|c|c|}
\hline $\mathrm{t}$ & Physical time [sec] \\
\hline $\mathrm{T}$ & Temperature [K] \\
\hline $\mathrm{U}$ & Vector of conservative variables \\
\hline UDF & User defined function \\
\hline URANS & Unsteady Reynolds-averaged Navier-Stokes \\
\hline \multicolumn{2}{|c|}{ Greek symbols } \\
\hline$\tau$ & Pseudo-time \\
\hline \multicolumn{2}{|c|}{ Subscript } \\
\hline $\mathrm{f}$ & fluid domain \\
\hline s & solid domain \\
\hline \multicolumn{2}{|c|}{ Superscript } \\
\hline $\mathrm{k}$ & Index of the sub-iteration \\
\hline $\mathrm{N}$ & Physical time step \\
\hline
\end{tabular}

1. Market Strategy Board White Paper; Energy Sources and use of Large-capacity Electrical Energy Storage. International Electrotechnical Commission, Switzerland, 2012. Retrieved September 19, 2014. http://www.iec.ch/whitepaper/gridintegration/?ref=extfooter (accessed August 17, 2017).

2. Fübi M, Felix Krull F, Ladwig Alstom M. A06 Flexibility Increase with Latest Technologies, VGB PowerTech Journal 3/2012.

3. Topel M, Jöcker M, Paul S, Laumert B. Differential Expansion Sensitivity Studies During Steam Turbine Startup. J Eng Gas Turbines Power 2015;138:62102. doi:10.1115/1.4031643.

4. Marinescu G, Ehrsam A. Experimental Investigation Into Thermal Behavior of Steam Turbine Components: Part 2-Natural Cooling of Steam Turbines and the Impact on LCF Life. ASME Turbo Expo 2012 Turbine Tech Conf Expo 2012;4:10. doi:10.1115/GT2012-68759.

5. Stein P, Marinescu G, Born D, Lerch M. Thermal Modeling and Mechanical Integrity Based Design of a Heat Shield on a High-Pressure Module Solar Steam Turbine Inner Casing With Focus on Lifetime. ASME Turbo Expo 2014 Turbine Tech Conf Expo 2014;5C:1-10. doi:10.1115/GT201425846.

6. Born D, Stein P, Marinescu G, Koch S, Schumacher D. Thermal Modelling of an Intermediate Pressure Steam Turbine by Means of Conjugate Heat Transfer: Simulation and Validation. Vol. 8 Microturbines, Turbochargers Small Turbomachines; Steam Turbines, ASME; 2016, p. V008T26A029. doi:10.1115/GT2016-57247. 
7. Mohr WF, Ruffino P. Experimental Investigation Into Thermal Behaviour of Steam Turbine Components: Part 1 - Temperature measurements with optical probes. ASME Turbo Expo 2012 Turbine Tech Conf Expo 2012;4:12. doi:10.1115/GT2012-68703.

8. Marinescu G, Mohr WF, Ehrsam A, Ruffino P, Sell M. Experimental Investigation Into Thermal Behavior of Steam Turbine Components - Temperature Measurements With Optical Probes and Natural Cooling Analysis. J Eng Gas Turbines Power 2013;136:10. doi:10.1115/1.4025556.

9. Marinecsu, G., Stein, P., Sell, M., Natural Cooling and Startup of Steam Turbines: Validity of the Over-Conductivity Function, Journal of Engineering for Gas Turbines and Power, November 2015, Vol. 137, 112601-1.

10. Maffulli, R., He, L., 2014, "Wall Temperature Effects on Heat Transfer Coefficient for High-Pressure Turbines,” J. Propuls. Power, 30(4), pp. 1080-1090. doi: 10.2514/1.B35126.

11. Sun Z, Chew JW, Hills NJ, Volkov KN, Barnes CJ. Efficient Finite Element Analysis/Computational Fluid Dynamics Thermal Coupling for Engineering Applications. J Turbomach 2010;132:31016. doi:10.1115/1.3147105.

12. Ganine V, Javiya U, Hills N, Chew J. Coupled Fluid-Structure Transient Thermal Analysis of a Gas Turbine Internal Air System With Multiple Cavities. J Eng Gas Turbines Power 2012;134:102508. doi:10.1115/1.4007060.

13. Errera MP, Baquué B. A quasi-dynamic procedure for coupled thermal simulations. Int J Numer Methods Fluids 2013;72:1183-206. doi:10.1002/fld.3782.

14. Wang Z, Corral R, Chaquet JM, Pastor G. Analysis and Improvement of a Loosely Coupled FluidSolid Heat Transfer Method. Proc. ASME TurboExpo, ASME; 2013, p. GT2013-94332. doi:10.1115/GT2013-94332.

15. Altaç Z, Uğurlubilek N. Assessment of turbulence models in natural convection from two- and threedimensional rectangular enclosures. Int J Therm Sci 2016;107:237-46. doi:10.1016/j.ijthermalsci.2016.04.016.

16. Ma J, Xu F. Transient Flows around a Fin at Different Positions. Procedia Eng., vol. 126, 2015, p. 393-8. doi:10.1016/j.proeng.2015.11.226.

17. Xu F, Saha SC. Transition to an unsteady flow induced by a fin on the sidewall of a differentially heated air-filled square cavity and heat transfer. Int J Heat Mass Transf 2014;71:236-44. doi:10.1016/j.ijheatmasstransfer.2013.12.019.

18. He L, Fadl M. Multi-scale time integration for transient conjugate heat transfer. Int J Numer Methods Fluids 2017;83:887-904. doi:10.1002/fld.4295.

19. Perelman TL. On conjugated problems of heat transfer. Into J Heat Mass Trans 1961; 3:293-303. doi:10.1016/0017-9310(61)90044-8. 
20. Jameson A. Time Dependent Calculations Using Multigrid, with Applications to Unsteady Flows Past Air foils and Wings. AIAA 10th Compute Fluid Din Cone 1991:14. doi:10.2514/6.1991-1596.

21. Arnone A. Integration of Navier-Stokes equations using dual time stepping and a multigrid method. AIAA J 1995; 33:985-90. doi:10.2514/3.12518.

22. He L. Three-dimensional unsteady Navier-Stokes analysis of stator-rotor interaction in axial-flow turbines. Proc Inst Mech Eng Part A J Power Energy 2000; 214:13-22. doi:10.1243/0957650001537813.

23. Fadl M, He L. On Large Eddy Simulation Based Conjugate Heat Transfer Procedure for Transient Natural Convection. ASME. J. Turbomach. 2017;139(11):111010-111010-9. doi:10.1115/1.4037492.

24. Fadl M, He L. On LES Based Conjugate Heat Transfer Procedure for Transient Natural Convection. ASME. Turbo Expo: Power for Land, Sea, and Air, Volume 5A: Heat Transfer :V05AT10A002. doi:10.1115/GT2017-63600

25. Fadl M, He L, Stein, P., Marinecsu, G., 'Assessment of Unsteadiness Modelling for Transient Natural Convection' ASME. Turbo Expo: Power for Land, Sea, and Air, Volume 8: Microturbines, Turbochargers and Small Turbomachines; Steam Turbines :V008T29A016. doi:10.1115/GT201763592.

26. Fadl M, He L, Stein, P., Marinecsu, G., 'Assessment of Unsteadiness Modelling for Transient Natural Convection’ ASME. J. Eng. Gas Turbines Power. 2017;140(1):012605-012605-10. doi:10.1115/1.4037721

27. Giles MB. Stability analysis of numerical interface conditions in fluid-structure thermal analysis. Int J Numer Methods Fluids 1997;25:421-36. doi:10.1002/(SICI)1097-0363(19970830)25:4

28. Chemieingenieurwesen V-Gv, Gesellschaft V, Fallis A. VDI Heat Atlas. vol. 53. Springer; 2010. doi:10.1017/CBO9781107415324.004. 